\title{
Estimation of Radial Power System Transfer Path Dynamic Parameters Using Synchronized Phasor Data
}

\author{
Joe H. Chow, Fellow, IEEE, Aranya Chakrabortty, Student Member, IEEE, Luigi Vanfretti, Student Member, IEEE, \\ and Murat Arcak, Senior Member, IEEE
}

\begin{abstract}
This paper develops a measurement-based method for estimating a two-machine reduced model to represent the interarea dynamics of a radial, multimachine power system. The method uses synchronized bus voltage phasor measurements at two buses and the line current on the power transfer path. The innovation is the application of the interarea oscillation components in the voltage variables resulting from disturbances for extrapolating system impedances and inertias beyond the measured buses. Expressions for the amplitudes of the bus voltage and bus frequency oscillations as functions of the location on the transmission path are derived from a small-signal perturbation approach. The reduced model provides approximate response to disturbances on the transfer path and offers an alternative to model reduction techniques based on detailed system models and data.
\end{abstract}

Index Terms-inertia extrapolation, interarea oscillations, phasor measurement, power system model reduction, power transfer interface, reactance extrapolation.

\section{INTRODUCTION}

$\mathbf{M}$ OST power system model reduction methods start from a detailed system model with a large parameter set. These methods are based on the observation that when subject to a disturbance, groups of close-by and strongly connected generators would swing coherently. Model reduction is then achieved by aggregating the coherent groups of generators together. These analytical reduction techniques include the terminal bus aggregation approach [1], [2] and the slow-coherency method [3]-[5]. An important attribute of these methods is that a power network structure is retained.

In this paper, we develop a measurement-based method for constructing a reduced physical model to represent the transfer path of a radial, multimachine power system, using bus voltage and line current phasors on the transfer path. The method is made practical by the availability of networks of GPS-synchronized, high sampling-rate (30 to 60 samples/s) phasor measurement units (PMU) [6], [7]. The innovation here

Manuscript received February 6, 2007; revised September 27, 2007. This research was supported in part by NSF under Grants ECS-0238268, ECS-0300025, and ECS-0622119, in part by the Power System Research Consortium Industry members (AEP, FirstEnergy, NYISO, and PJM), and in part by the Rensselaer Center for Automation Technologies and Systems supported by NYSTAR. Paper no. TPWRS-00070-2007.

The authors are with the Electrical, Computer, and Systems Engineering Department, Rensselaer Polytechnic Institute, Troy, NY 12180-3590 USA.

Color versions of one or more of the figures in this paper are available online at http://ieeexplore.ieee.org.

Digital Object Identifier 10.1109/TPWRS.2008.919315 is the application of the interarea oscillation components in the voltage variables resulting from disturbances for extrapolating system impedances and inertias beyond the measured buses. Starting from a small-signal model, fundamental expressions for the amplitudes of the bus voltage and bus frequency oscillations as functions of the location on the transmission path of a two-machine system are derived. This concept is then extended to obtain the impedance and inertia of a coherent area in a radial power system, by extracting the interarea model oscillations. The results here extend those in [8], where only the impedance between the measured buses and an equivalent inertia were computed. The work here differs from several out-of-step relay studies [9], [10] which rely on known or precomputed machine and system reactances.

Dynamic analysis of synchronized phasor data obtained in the U.S. west coast system from disturbances and staged tests has focused on low-frequency interarea damping calculation [11], transfer function analysis [11], and the validation of system models and parameters [13]. This paper provides a new application of phasor measurement data.

The remainder of this paper is organized as follows. Section II poses the reduced interarea model estimation problem using a two-area power system as an illustration. Section III discusses the reactance and inertia extrapolation algorithms for a twomachine system. Section IV illustrates the extrapolation algorithms using a two-machine system with voltage regulator control. Section V extends the extrapolation algorithms to a twoarea power system. The disturbance response of the reduced interarea model is shown in Section VI. The application of the method to a transfer path in the WECC system using actual measured data is provided in Section VII.

\section{PRoblem Formulation}

Consider the two-area power system with four machines and 13 buses (see Fig. 1) modified from the one used in [14] and [15]. The data for this system are given in Appendix A. In this system, Generators $G_{11}$ and $G_{12}$ are strongly connected and Generators $G_{21}$ and $G_{22}$ are also strongly connected. The connections between Generators $\left(G_{11}, G_{12}\right)$ and Generators $\left(G_{21}, G_{22}\right)$, however, are weaker. As a result, $G_{11}$ and $G_{12}$ form a coherent group or area and $G_{21}$ and $G_{22}$ form a second coherent group. For a disturbance external to a coherent group, the generators in the coherent group will swing in unison with each other and against other coherent groups of generators. Such interarea modes of 


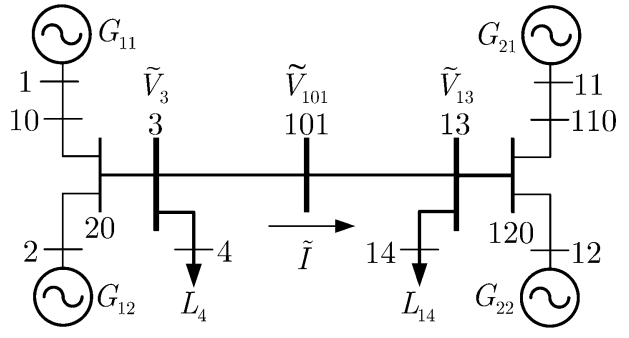

Fig. 1. Two-area power system.

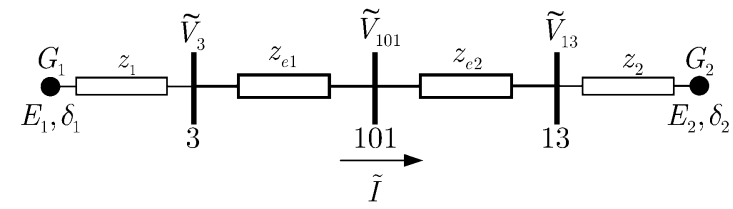

Fig. 2. Reduced model of the two-area system.

oscillations are lower in frequency than the local mode of oscillations between machines in the same coherent area. For example, with $300 \mathrm{MW}$ power transfer from Area 1 to Area 2, the interarea oscillation frequency is $0.5735 \mathrm{~Hz}$, and the local mode frequencies within the coherent areas are $1.2930 \mathrm{~Hz}$ and 1.3076 $\mathrm{Hz}$.

This two-area system is useful for representing a radial transfer path in a large power system in which one coherent area is exporting power to the other coherent area. In a realistic system, there will be many more generators in each coherent area. With an aggregate machine representing each coherent group, we rationalize that there is a center point in an equivalent machine where the voltage magnitude is fixed during a disturbance. Let these fixed voltages be $\tilde{E}_{1}=E_{1} \varepsilon^{j \delta_{1}}$ and $\tilde{E}_{2}=E_{2} \varepsilon^{j \delta_{2}}$ for Areas 1 and 2, respectively. Thus, the transfer path can be represented by a reduced-order model consisting of impedances connecting to the center points of the coherent areas, as shown in Fig. 2, which are the internal voltage nodes of the equivalent generators $G_{1}$ and $G_{2}$, with inertias $H_{1}$ and $\mathrm{H}_{2}$, respectively. Note that Buses 3,13, and 101 are retained in the reduced model, and the total impedance between Buses 3 and 13 is $z_{e}=z_{e 1}+z_{e 2}$. Thus, the two-area power system in Fig. 1 is considered as having a radial transfer path.

In this paper, we develop a method to construct a reducedorder dynamic model of the transfer path, using synchronized voltage phasor measurement data at Buses 3 and $13\left(\tilde{V}_{3}=\right.$ $V_{3} \varepsilon^{j \theta_{3}}$ and $\tilde{V}_{13}=V_{13} \varepsilon^{j \theta_{13}}$, respectively) and the current phasor from Bus 3 to Bus $13\left(\tilde{I}=I \varepsilon^{j \theta_{I}}\right)$. We pose the problem of finding the reduced model in Fig. 2 as follows.

Interarea Model Estimation (IME) Problem: Given the measured synchronized phasor variables $V_{3}, \theta_{3}, V_{13}, \theta_{13}, I$, and $\theta_{I}$ in Fig. 1 that exhibit a few cycles of interarea oscillations, compute $E_{i}, \delta_{i}, z_{i}$, and $H_{i}, i=1,2$, and $z_{e}$ of the reduced-order two-machine system in Fig. 2 to represent the interarea dynamic behavior of the two-area power system.

The IME problem can be generalized to handle two-area systems whose transfer interfaces consist of multiple lines. In such cases, phasor measurement units need to be installed to measure

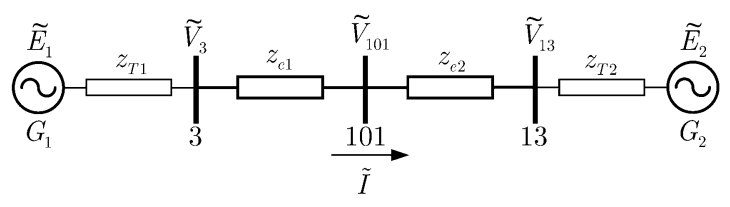

Fig. 3. Two-machine power system.

the voltages at all the buses at the sending and receiving ends of the interfaces and the currents across the interface. To solve the IME problem, we first develop a method to find the parameters of a two-machine system based on the phasor measurements recorded during a disturbance. Then the reduced system model in Fig. 2 is constructed using only the interarea oscillations between the two areas.

\section{Dynamic Model Estimation FOR A TWO-MACHINE SYSTEM}

Consider the two-machine power system in Fig. 3 where the inertias and the internal voltage phasors of the generators $G_{1}$ and $G_{2}$ are $H_{1}$ and $H_{2}$, and $\tilde{E}_{1}$ and $\tilde{E}_{2}$, respectively. We assume that $G_{1}$ is supplying power to $G_{2}$, which acts as a load. The generators are connected to Buses 3 and 13 through transformers having impedances $z_{T 1}=r_{T 1}+j x_{T 1}$ and $z_{T 2}=r_{T 2}+j x_{T 2}$, respectively. The line current phasor $\tilde{I}$ flowing from Bus 3 to Bus 13 is $I=I \varepsilon^{j \theta_{I}}$. Bus 101 is located between Buses 3 and 13. The impedances connecting the generator internal voltages to Buses 3 and 13 are thus given by

$$
z_{i}=r_{T i}+j\left(x_{T i}+x_{d i}^{\prime}\right)=r_{i}+j x_{i}, i=1,2
$$

where $x_{d 1}^{\prime}$ and $x_{d 2}^{\prime}$ are either the direct-axis transient or subtransient reactances of $G_{1}$ and $G_{2}$, respectively, depending on the generator models. The internal node model of the system in Fig. 3 is also represented by Fig. 2.

The dynamic model of the two-machine system in Fig. 3, neglecting damping, is given by

$$
\dot{\delta}_{i}=\Omega \omega_{i}, \quad 2 H_{i} \dot{\omega}_{i}=P_{m i}-P_{e i}, \quad i=1,2
$$

where $\omega_{i}$ is the speed, and $P_{m i}$ and $P_{e i}$ are the mechanical power input and electrical power output, respectively, of the $i$ th machine. The constant $\Omega$ is the conversion factor from p.u. speed to $\mathrm{rad} / \mathrm{s}$. The two swing equations (2) can be combined to form a second-order system

$$
\dot{\delta}=\Omega \omega, \quad 2 H \dot{\omega}=P_{m}-P_{e}-P_{\text {loss }}
$$

with

$$
\begin{aligned}
P_{m} & =\frac{H_{2} P_{m 1}-H_{1} P_{m 2}}{H_{s}} \\
P_{\text {loss }} & =\frac{r_{e}^{\prime}}{z_{m}} \frac{H_{2} E_{1}^{2}+H_{1} E_{2}^{2}}{H_{s}} \\
P_{e} & =-\frac{E_{1} E_{2}}{z_{m}} \frac{H_{2} \cos (\delta+\alpha)-H_{1} \cos (\delta-\alpha)}{H_{s}}
\end{aligned}
$$

where $P_{\text {loss }}$ represents the losses, $z_{e}^{\prime}=z_{e 1}+z_{e 2}+z_{1}+z_{2}=$ $z_{m} \varepsilon^{j \alpha}$ is the total line impedance, $r_{e}^{\prime}=\operatorname{Re}\left\{z_{e}^{\prime}\right\}$ is the total line 


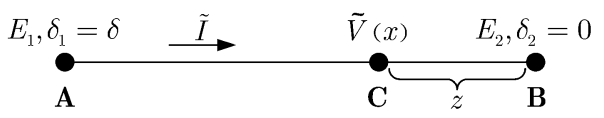

Fig. 4. Two-machine power system line diagram.

resistance, $H=H_{1} H_{2} /\left(H_{1}+H_{2}\right)$ is the equivalent inertia, $H_{s}=H_{1}+H_{2}, \delta=\delta_{1}-\delta_{2}$, and $\omega=\omega_{1}-\omega_{2}$.

We assume that phasor measurement units are located at Buses 3 and 13, and synchronized phasor variables $\tilde{V}_{3}, \tilde{V}_{13}$, and $\tilde{I}$ as a result of a disturbance are available. Based on the measured data, one of these quantities, namely, $z_{e}$, can readily be computed as

$$
z_{e}=\left(\tilde{V}_{3}-\tilde{V}_{13}\right) / \tilde{I} .
$$

In addition, if $z_{1}$ and $z_{2}$ are known, then the machine internal voltages can be computed as

$$
\tilde{E}_{1}=\tilde{V}_{3}+j z_{1} \tilde{I}, \quad \tilde{E}_{2}=\tilde{V}_{13}-j z_{2} \tilde{I} .
$$

Thus, the problem of estimating the two-machine power system model reduces to the estimation of $z_{1}$ and $z_{2}$, and the inertias $H_{1}$ and $H_{2}$. They will be computed using extrapolation techniques described in the following subsections.

We remark that if the voltage and current phasor measurements are available at only one bus, say Bus 3 , the dynamic model can still be estimated if the impedance $z_{e}=z_{e 1}+z_{e 2}$ from Bus 3 to Bus 13 is known. In this case, the voltage phasor at Bus 13 can be computed from the expression (5).

\section{A. Reactance Extrapolation Algorithm}

To the dynamic model (3), we will append an expression for the voltage at any point along the total impedance $z_{e}^{\prime}=z_{1}+$ $z_{e}+z_{2}=r_{e}^{\prime}+j x_{e}^{\prime}$, as illustrated by Fig. 4, where the two endpoints $\mathrm{A}$ and $\mathrm{B}$ represent the internal nodes of $G_{1}$ and $G_{2}$. $\mathrm{C}$ is any point on the line between $\mathrm{A}$ and $\mathrm{B}$, at an impedance of $z=r+j x$ from $\mathrm{B}$.

Given the line current phasor $\tilde{I}$, the voltage phasor at the point $\mathrm{C}$ is

$$
\begin{aligned}
\tilde{V}(x, r)= & \tilde{E}_{2}+(r+j x) \tilde{I} \\
= & E_{2}+\frac{r+j x}{r_{e}^{\prime}+j x_{e}^{\prime}}\left(E_{1} \cos (\delta)\right. \\
& \left.+j E_{1} \sin (\delta)-E_{2}\right) \\
= & {\left[E_{2}(1-a)+E_{1}(a \cos (\delta)-b \sin (\delta))\right] } \\
& +j\left[E_{1}(b \cos (\delta)+a \sin (\delta))-b E_{2}\right]
\end{aligned}
$$

where

$$
a=\frac{r r_{e}^{\prime}+x x_{e}^{\prime}}{{r_{e}^{\prime}}^{2}+x_{e}^{\prime 2}}, \quad b=\frac{x r_{e}^{\prime}-r x_{e}^{\prime}}{{r_{e}^{\prime 2}}^{2}+x_{e}^{\prime 2}}
$$

The magnitude of $\tilde{V}(x)$ is, therefore, calculated as (9) at the bottom of the page, where $c=E_{2}^{2}\left(b^{2}+(1-a)^{2}\right)+E_{1}^{2}\left(a^{2}+b^{2}\right)$.

Linearizing (3) and (9) about an equilibrium point $\left(\delta_{0}, \omega_{0}=\right.$ $\left.0, V_{s s}\right)$, we obtain

$$
\begin{aligned}
\Delta \dot{\delta}= & \Omega \Delta \omega \\
2 H \Delta \dot{\omega}= & \frac{E_{1} E_{2}}{z_{m} H_{s}}\left(H_{2} \sin \left(\delta_{0}+\alpha\right)\right. \\
& \left.-H_{1} \sin \left(\delta_{0}-\alpha\right)\right) \Delta \delta \\
\Delta V(x)= & J\left(a, b, \delta_{0}\right) \Delta \delta
\end{aligned}
$$

where the Jacobian $J\left(a, b, \delta_{0}\right)$ is

$$
\begin{aligned}
J\left(a, b, \delta_{0}\right) & =\left.\frac{\partial V(a, b, \delta)}{\partial \delta}\right|_{\delta=\delta_{0}} \\
& =\frac{-E_{1} E_{2}\left(\sin \left(\delta_{0}\right)\left(a-a^{2}-b^{2}\right)+b \cos \left(\delta_{0}\right)\right)}{V\left(a, b, \delta_{0}\right)} .
\end{aligned}
$$

Of prime importance is that $J\left(a, b, \delta_{0}\right)(11)$ consists of two parts: a numerator part varying as $a$ and $b$, and a denominator part which is the steady-state bus magnitude at the location C. Note that there is no explicit assumption on the relationship between $r$ and $x$ in the derivation of (7)-(11). The ratio $r / x$ may vary from point $\mathrm{A}$ to point $\mathrm{B}$.

If the resistance is uniform between $\mathrm{A}$ and $\mathrm{B}$, such that

$$
r / x=r_{e}^{\prime} / x_{e}^{\prime}
$$

which includes the case where the resistance $r_{e}^{\prime}$ is negligible, (8) reduces to $a=x / x_{e}^{\prime}, b=0$. As a result, $J\left(a, b, \delta_{0}\right)$ reduces to

$$
J\left(a, 0, \delta_{0}\right)=-E_{1} E_{2} \sin \left(\delta_{0}\right) \frac{(1-a) a}{V\left(a, 0, \delta_{0}\right)} .
$$

The plot of $a(1-a)$ from $a=0$ (point A) to $a=1$ (point $\mathrm{B}$ ) is shown as the solid curve in Fig. 5. ${ }^{1}$ Note that it closely resembles a sine function from $0^{\circ}$ to $180^{\circ}$, denoted by the dotted curve. ${ }^{2}$

Suppose that the two-machine system is subject to a disturbance, inducing an oscillation on the bus voltages. Then $J(11)$ can be used to compute the amplitude of the voltage oscillation at various locations between the two machines, provided that the oscillations remain largely linear and $E_{1}, E_{2}$, and $\delta_{0}$ are known.

\footnotetext{
${ }^{1}$ The Jacobian fit can accommodate series-compensated lines. When a series capacitor $-j x_{c}$ is encountered, we move to the left by an amount normalized for $-x_{c}$.

${ }^{2}$ The sinusoidal representation can be justified by treating the reactance $x$ as a distributed parameter. Hence, the voltage wave generated along the line following a perturbation is given as the solution of a wave equation of the form $\partial^{2} V / \partial t^{2}=\gamma^{2} \partial^{2} V / \partial x^{2}$, where $\gamma$ is propagation velocity. The solution consists of a forward traveling wave and a backward traveling wave so that a fixed time point, the voltage wave can be written as a standing wave $V(x)=$ $A \sin (2 \pi x / \lambda)$ of amplitude $A$, where $\lambda$ is the wavelength of the traveling waves.
}

$$
\begin{aligned}
V(x, r) & =|\tilde{V}(x, r)| \\
& =\sqrt{c+2 E_{1} E_{2}\left(\left(a-a^{2}-b^{2}\right) \cos (\delta)-b \sin (\delta)\right)} \\
& =V(a, b, \delta)
\end{aligned}
$$




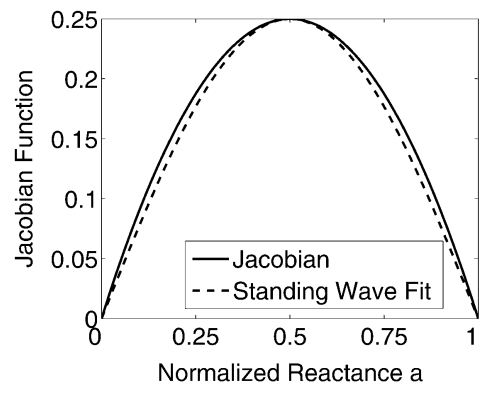

Fig. 5. Comparison of Jacobian fit (solid) and standing wave fit (dashed).

Furthermore, if the voltage oscillations are measured or calculated at Bus 101, we can use (11) to compute $z_{1}$ and $z_{2}$.

We will illustrate the computation of $z_{1}$ and $z_{2}$ for the uniform resistance case. The general case can also be computed, but the resulting expressions are more involved. Following the disturbance, the amplitudes of the voltage oscillations at these three buses are measured as $V_{3 m}, V_{13 m}$, and $V_{101 m}$. If Bus 101 does not physically exist, we can take it to be the midpoint between Buses 3 and 13, such that

$$
\tilde{V}_{101}=\tilde{V}_{3}-j z_{e 1} \tilde{I}=\tilde{V}_{3}-j\left(z_{e} / 2\right) \tilde{I} .
$$

Using (13), we obtain three normalized voltage equations

$$
V_{\mathrm{in}}=V_{i m} V_{\mathrm{iss}}=A\left(1-a_{i}\right) a_{i}, \quad i=3,13,101
$$

where $A=-E_{1} E_{2} \sin \left(\delta_{0}\right)$ and $V_{\text {iss }}$ is the steady-state value of $\tilde{V}_{i}$. These three values $V_{3 n}, V_{13 n}$, and $V_{101 n}$ will uniquely define the curve $E_{1} E_{2} \sin \left(\delta_{0}\right)(1-a) a$.

Note that the three equations in (15) contain three unknowns $A$, and $x_{1}$ and $x_{2}$, which determine $a_{3}, a_{13}$, and $a_{101}$ as $a_{13}=$ $x_{2} /\left(x_{1}+x_{e}+x_{2}\right), a_{101}=\left(x_{2}+x_{e} / 2\right) /\left(x_{1}+x_{e}+x_{2}\right)$ and $a_{3}=\left(x_{2}+x_{e}\right) /\left(x_{1}+x_{e}+x_{2}\right)$. The constant $A$ can be eliminated by dividing two of the equations in (15) by the third equation, such that

$$
\begin{aligned}
V_{3 n}\left(1-a_{101}\right) a_{101} & =V_{101 n}\left(1-a_{3}\right) a_{3} \\
V_{13 n}\left(1-a_{101}\right) a_{101} & =V_{101 n}\left(1-a_{13}\right) a_{13}
\end{aligned}
$$

which can be solved for $x_{1}$ and $x_{2}$ using a nonlinear equation solver.

For systems where $r_{1}$ and $r_{2}$ are nonzero and the ratios $r_{1} / x_{1}$ and $r_{2} / x_{2}$ are not known, two more measurements between Buses 3 and 13 are needed.

\section{B. Inertia Extrapolation Algorithm}

Once $x_{1}$ and $x_{2}$ have been computed, the remaining parameters to be computed are the inertias $H_{1}$ and $H_{2}$. We need two pieces of information. First, from the linearized model (10), the inter-area frequency (in $\mathrm{Hz}$ ) is given as

$$
f=\frac{1}{2 \pi} \sqrt{\frac{E_{1} E_{2} \Omega\left(H_{2} \sin \left(\delta_{0}+\alpha\right)-H_{1} \sin \left(\delta_{0}-\alpha\right)\right)}{2 H_{s} H z_{m}}} .
$$

Thus, by measuring the frequency $f$ of the swing mode in the voltage measurement, the equivalent inertia constant $H$ can be computed as

$$
H=\frac{E_{1} E_{2} \Omega\left(H_{2} \sin \left(\delta_{0}+\alpha\right)-H_{1} \sin \left(\delta_{0}-\alpha\right)\right)}{2 H_{s} z_{m}(2 \pi f)^{2}} .
$$

When the line resistance is negligible compared to the reactance, then $\alpha \approx 90^{\circ}$, and hence, (19) can be reduced to

$$
H=\frac{E_{1} E_{2} \cos \left(\delta_{0}\right) \Omega}{2 x_{e}^{\prime}(2 \pi f)^{2}} .
$$

Second, to calculate $H_{1}$ and $H_{2}$ separately, we develop a companion equation by exploiting the frequencies measured at Buses 3 and 13. Neglecting losses and machine damping effects, the conservation of the total angular momentum of the two-machine system is given as

$$
\begin{aligned}
L & =2 H_{1} \omega_{1}+2 H_{2} \omega_{2}=2 \int\left(H_{1} \dot{\omega}_{1}+H_{2} \dot{\omega}_{2}\right) d t \\
& =\int\left(P_{m 1}-P_{e 1}+P_{m 2}-P_{e 2}\right) d t=0
\end{aligned}
$$

from which we obtain

$$
\frac{H_{1}}{H_{2}}=-\frac{\omega_{2}}{\omega_{1}} .
$$

Hence, (22) and (19) together can be used to solve for $H_{1}$ and $\mathrm{H}_{2}$, provided that the estimates for the machine speeds are known. We next show that $\omega_{1}$ and $\omega_{2}$ can indeed be estimated from the measured frequencies $\vartheta_{3}$ and $\vartheta_{13}$ at Buses 3 and 13 .

For the two-machine system, we can write

$$
\tilde{V}_{3}=\tilde{E}_{1}-\left(j x_{1}\right) \tilde{I}=\tilde{E}_{1}-\rho_{1}\left(\tilde{E}_{1}-\tilde{E}_{2}\right)
$$

where $\rho_{1}=x_{1} / x_{e}^{\prime}$. Thus, the voltage angle at Bus $3, \theta_{3}$, can be expressed as $\tan \left(\theta_{3}\right)=\operatorname{Im}\left(\tilde{V}_{3}\right) / \operatorname{Re}\left(\tilde{V}_{3}\right)$. Differentiating $\theta_{3}$ with respect to time $t$, we get

$$
\sec ^{2}\left(\theta_{3}\right) \dot{\theta}_{3}=\frac{d}{d t}\left(\tan \theta_{3}\right)=\Omega \frac{\phi_{1}}{\phi_{2}}
$$

where

$$
\begin{aligned}
\phi_{1}= & \omega_{1} E_{1}^{2}\left(1-\rho_{1}\right)^{2} \\
& +E_{1} E_{2} \rho_{1}\left(1-\rho_{1}\right) \cos \left(\delta_{1}-\delta_{2}\right)\left(\omega_{1}+\omega_{2}\right) \\
& +\omega_{2} r_{1}^{2} E_{2}^{2} \\
\phi_{2}= & \left(E_{1} \cos \left(\delta_{1}\right)-\rho_{1} E_{1} \cos \left(\delta_{1}\right)+\rho_{1} E_{2} \cos \left(\delta_{2}\right)\right)^{2} .
\end{aligned}
$$

The expressions in (24) can be readily derived from the dynamic model (2) for the two-machine machine. Also, deriving the expression for $\sec \left(\theta_{3}\right)$ from $\tan \left(\theta_{3}\right)$ and using it in (24), we can show that the frequency derivation $\vartheta_{3}=\dot{\theta}_{3} / \Omega$ in p.u. from nominal frequency at Bus 3 is given as

$$
\vartheta_{3}=\frac{g_{1} \omega_{1}+h_{1}\left(\omega_{1}+\omega_{2}\right) \cos \left(\delta_{1}-\delta_{2}\right)+k_{1} \omega_{2}}{g_{1}+2 h_{1} \cos \left(\delta_{1}-\delta_{2}\right)+k_{1}}
$$

where $g_{1}=E_{1}^{2}\left(1-\rho_{1}\right)^{2}, h_{1}=E_{1} E_{2} \rho_{1}\left(1-\rho_{1}\right)$, and $k_{1}=$ $\rho_{1}^{2} E_{2}^{2}$. 


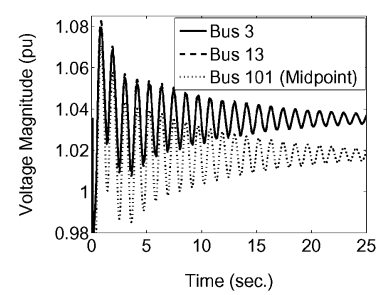

(a)

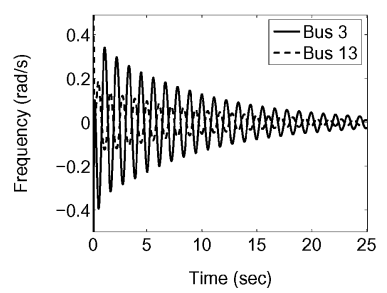

(b)
Fig. 6. Voltage and frequency oscillations at three buses in the two-machine system with AVR. (a) Voltage magnitude. (b) Bus frequency.

Similarly, the frequency deviation at Bus 13 is

$$
\vartheta_{13}=\frac{\dot{\theta}_{13}}{\Omega}=\frac{g_{2} \omega_{1}+h_{2}\left(\omega_{1}+\omega_{2}\right) \cos \left(\delta_{1}-\delta_{2}\right)+k_{2} \omega_{2}}{g_{2}+2 h_{2} \cos \left(\delta_{1}-\delta_{2}\right)+k_{2}}
$$

where $g_{2}=E_{1}^{2}\left(1-\rho_{2}\right)^{2}, h_{2}=E_{1} E_{2} \rho_{2}\left(1-\rho_{2}\right)$, and $k_{2}=$ $\rho_{2}^{2} E_{2}^{2}$, with $\rho_{2}=\left(x_{1}+x_{e}\right) / x_{e}^{\prime}$.

Because the bus frequencies $\vartheta_{3}$ and $\vartheta_{13}$ are available from synchronous phasor measurements, we can estimate $\omega_{1}$ and $\omega_{2}$ using (26) and (27), calculate the ratio $\omega_{2} / \omega_{1}=-H_{1} / H_{2}$, and solve for $H_{1}$ and $H_{2}$ using (20).

\section{Two-Machine System With Detailed Machine MODEL EQUIPPED WITH AVR}

In this section, we will use the algorithms in Section III to compute the dynamic model of the two-machine system in Fig. 3, with detailed machine models equipped with automatic voltage regulators (AVRs). Both machine models include subtransient effects, and a type AC4A [16] voltage regulator model is used for both machines. The parameters of this system are given in Appendix B. Note that there is no fixed voltage source in the subtransient model, because the voltages within the synchronous machine are controlled by the AVR. However, it is still useful to conceptualize that there is a fixed voltage source, albeit fictitious, inside the machine, as impacted by the AVR.

A disturbance is applied to the system and the resulting bus voltage magnitude response, simulated using the MATLAB Power System Toolbox [17], is shown in Fig. 6(a). Note the interarea mode oscillates at a frequency of $0.909 \mathrm{~Hz}$. Furthermore, the voltage magnitude swings at Buses 3 and 13 are in phase.

From the voltage response, we obtain the normalized voltage oscillation amplitudes for fitting the Jacobian function as (with the negative sign dropped)

$$
V_{3 n}=0.0215, \quad V_{13 n}=0.0234, \quad V_{101 n}=0.0273 \text {. }
$$

From (16) and (17), we obtain the values of $x_{1}$ and $x_{2}$ in Table I. The Jacobian curve fit of the reactance results is shown in Fig. 7(a).

To calculate $H_{1}$ and $H_{2}$, we compute bus frequencies $\vartheta_{3}$ and $\vartheta_{13}$ by passing the bus voltage angles $\theta_{3}$ and $\theta_{13}$ through a derivative filter $G(s)=s /(T s+1)$, where the time constant $T$ is $=0.02 \mathrm{~s}$. The bus frequencies $\vartheta_{3}$ and $\vartheta_{13}$ are shown in Fig. $6(\mathrm{~b})$. It is important to note that the frequency oscillations are out of phase. The estimated initial parameters are shown in Table I.

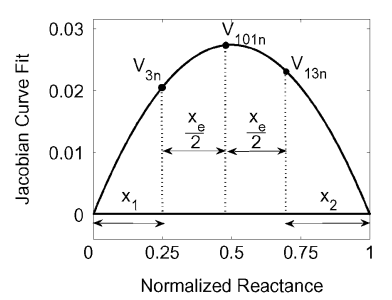

(a)

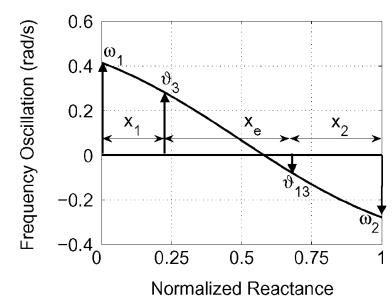

(b)
Fig. 7. Reactance and inertia extrapolations. (a) Voltage magnitude fit. (b) Frequency fit.

TABLE I

ESTIMATED PARAMETERS FOR DETAILED SYSTEM MODEL (900-MVA BASE)

\begin{tabular}{|c|c|}
\hline Parameters & Estimated Values \\
\hline$x_{1}$ & $0.3142 \mathrm{pu}$ \\
$x_{2}$ & $0.3594 \mathrm{pu}$ \\
$E_{1}$ & $1.105 \mathrm{pu}$ \\
$\delta_{1}$ & $37.08^{\circ}$ \\
$E_{2}$ & $1.118 \mathrm{pu}$ \\
$\delta_{2}$ & $-10.27^{\circ}$ \\
$H$ & $4.110 \mathrm{pu}$ \\
$H_{1}$ & $6.600 \mathrm{pu}$ \\
$H_{2}$ & $10.710 \mathrm{pu}$ \\
\hline
\end{tabular}

The extrapolation of the machine speeds from the bus frequencies is illustrated in Fig. 7(b).

It is of interest to note that the estimated values of $x_{1}$ and $x_{2}$ satisfy

$$
\begin{aligned}
& x_{1}-x_{T 1}=x_{\mathrm{VR} 1}=0.2242 \mathrm{pu}>x_{d 1}^{\prime \prime} \\
& x_{2}-x_{T 2}=x_{\mathrm{VR} 2}=0.2694 \mathrm{pu}>x_{d 2}^{\prime \prime} .
\end{aligned}
$$

Thus, we view that the AVRs move the fixed voltage points from the voltages behind the subtransient reactances to be further behind the reactances $x_{\mathrm{VR} 1}$ and $x_{\mathrm{VR} 2}$.

\section{ILLUSTRATION With Two-AREA SySTEM}

In this section, we extend the algorithms in Section III to find the reduced IME model parameters of the two-area system in Fig. 1 for several power transfer levels from Area 1 to Area 2. For the case with a power transfer of $300 \mathrm{MW},{ }^{3}$ we apply a disturbance to the two-area system and simulate the system response using [17]. The disturbance is a three-phase fault at Bus 101 cleared after $0.05 \mathrm{~s}$ without any line switchings. The voltage phasors $\tilde{V}_{3}$ and $\tilde{V}_{13}$ and the current phasor $\tilde{I}$ flowing from Bus 3 to Bus 13 are measured. The magnitude of these quantities are shown in Fig. 8. Note that the Bus 101 voltage phasor can be either measured or computed from (14).

Because this two-area system has four machines, there are three swing modes, one of which is the interarea mode at 0.5735 Hz. From Fig. 8, the interarea mode oscillation is dominant in all three voltages. We apply the Eigensystem Realization Algorithm (ERA) to extract the modes and their mode shapes in the time response [18], [19], using the time period of 1 to $10 \mathrm{~s}$ to avoid the initial voltage transients. ${ }^{4}$ In addition to the steady-state values, ERA shows that the oscillations can be approximated by two modes, a $0.5732-\mathrm{Hz}$ interarea mode and a $1.0823-\mathrm{Hz}$ local mode. Fig. 9 shows the $0.5732-\mathrm{Hz}$ mode

\footnotetext{
${ }^{3}$ Due to losses, the actual power transfer is about $339 \mathrm{MW}$.

${ }^{4}$ The Prony method [12] can also be used.
} 


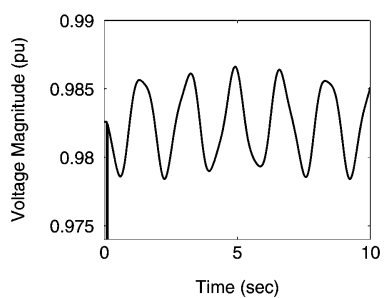

(a)

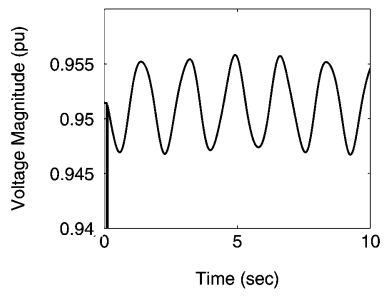

(c)

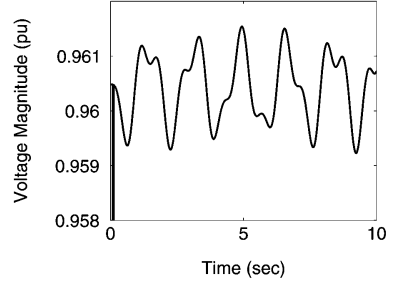

(b)

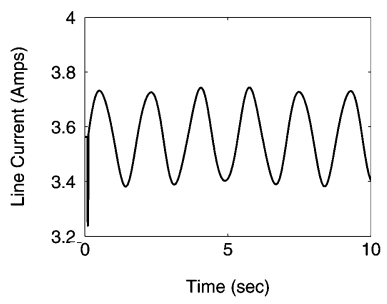

(d)
Fig. 8. Simulation results for two-area system. (a) Bus 3 voltage magnitude. (b) Bus 13 voltage magnitude. (c) Bus 101 voltage magnitude. (d) Line current magnitude.

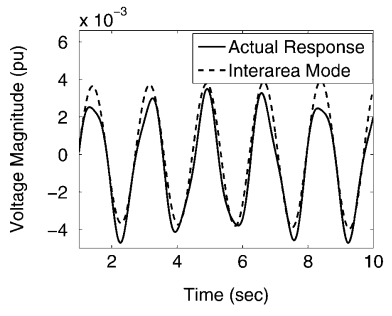

(a)

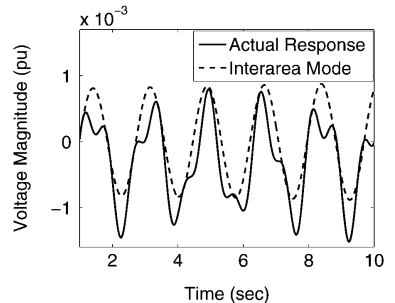

(b)

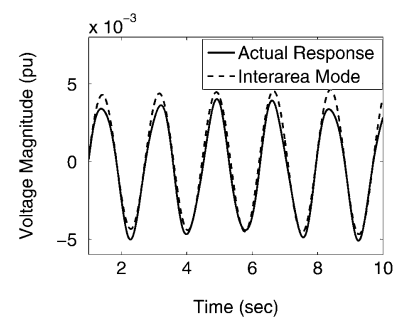

(c)

Fig. 9. Two-mode approximation of the voltage oscillation with the steadystate shifted to zero. (a) Bus 3 voltage magnitude. (b) Bus 13 voltage magnitude. (c) Bus 101 voltage magnitude.

components superimposed on the individual voltage magnitude oscillations. The normalized voltage amplitudes are found to be

$$
\begin{gathered}
V_{3 n}=0.0036456, \quad V_{13 n}=0.0007939 \\
V_{101 n}=0.004166 .
\end{gathered}
$$

Using the reactance and inertia extrapolation algorithms, the parameters of the reduced IME model are shown in Table II.

Note that although the network impedances in one area are a mirror image of the other area, the estimated values of $x_{1}$ and $x_{2}$ are quite different, with $x_{1}$ almost eight times larger than $x_{2}$. This difference is attributed to the different loads in the areas. In Area 2, a current with a magnitude of 14.36 p.u. flowing from the two generators in Area 2 on Line 120-13 is actually opposite in direction to the flow of the current $\tilde{I}$. Hence, $x_{2}$ is expected to be smaller. Also, in the reduced IME model, $H_{1}$ is larger than
TABLE II

IME MODEL PARAMETERS FOR DIFFERENT POWER TRANSFER LEVElS (900-MVA BASE)

\begin{tabular}{|c|c|c|c|}
\hline \multirow{2}{*}{ Parameters } & \multicolumn{3}{|c|}{ Estimated Values } \\
\cline { 2 - 4 } & $150 \mathrm{MW}$ & $300 \mathrm{MW}$ & $450 \mathrm{MW}$ \\
\hline$x_{1}$ & 0.7613 & 0.5069 & 0.4154 \\
$x_{2}$ & -0.0749 & 0.0618 & 0.0897 \\
$E_{1}$ & 1.010 & 1.034 & 1.053 \\
$\delta_{1}$ & $3.37^{\circ}$ & $5.57^{\circ}$ & $7.39^{\circ}$ \\
$E_{2}$ & 0.985 & 0.966 & 0.946 \\
$\delta_{2}$ & $-12.91^{\circ}$ & $-30.24^{\circ}$ & $-39.24^{\circ}$ \\
$f$ & 0.6045 & 0.5732 & 0.5503 \\
$H$ & 7.44 & 7.555 & 7.221 \\
$H_{1}$ & 15.58 & 18.98 & 25.34 \\
$H_{2}$ & 14.24 & 12.55 & 10.09 \\
\hline
\end{tabular}

the sum of the individual machine inertias in Area 1, whereas $\mathrm{H}_{2}$ is smaller than the sum of individual machines in Area 2.

To show the robustness of this measurement-based model reduction approach, we studied two more cases. We applied the same three-phase fault at Bus 101 but cleared the fault after 0.1 $\mathrm{s}$, and we applied a three-phase fault at Bus 13 of the two-area system, cleared after $0.05 \mathrm{~s}$ both without any line switching. The resulting values of $x_{1}, x_{2}, H_{1}$, and $H_{2}$ were practically identical to those obtained for the first disturbance.

From the earlier discussion, the IME model will depend on the amount of power transfer between the two areas. The reactance estimation is also performed for power transfer levels of $150 \mathrm{MW}$ and $450 \mathrm{MW}$ from Area 1 to Area 2, with the resulting model parameters given in Table II. These power transfer levels are achieved by adjusting the loads on Buses 4 and 14, while keeping the total load to be the same.

As expected, Table II shows that as the power transfer increases to $450 \mathrm{MW}, x_{1}$ decreases and $x_{2}$ increases. The angular difference $\delta_{1}-\delta_{2}$ also increases because of the higher loading level. The equivalent inertia computation shows that $H_{1}$ becomes higher and $H_{2}$ becomes smaller. Note that for the 150-MW transfer case, the interarea mode component of the voltage oscillation at Bus 13 is out of phase with that at Bus 3 . As a result, $x_{2}$ is negative. This implies that the fixed voltage point is between Buses 3 and 13, and not beyond Bus 13, as in the $300 \mathrm{MW}$ and $450 \mathrm{MW}$ transfer cases. Such situations with negative values of $x_{2}$ are not unexpected for transfer paths at very low power transfer levels, when the interarea mode component is not dominant. This should not be viewed as a shortcoming of this approach because at low transfer levels, the interconnected power system has a substantial stability margin and there is little need for a reduced interarea model aimed at stability margin analysis. Furthermore, this analysis shows that for real-time applications, the flow on the transfer path and the reactance of the transfer path must be known for choosing the appropriate IME model.

\section{Disturbance Simulation Using The IME Model}

The main purpose of obtaining the reduced IME model from synchronized phasor data is to use it for predicting system stability. To this end, we study the disturbance responses of the full model, a reduced model obtained from the inertia-aggregation (INA) technique, [3] $]^{5}$ and the IME model.

\footnotetext{
${ }^{5}$ The impedances of the reduced model are obtained by paralleling the impedances of the coherent generators connected to the same aggregate bus.
} 


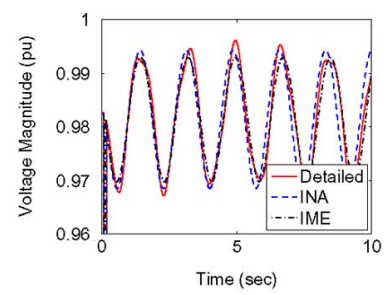

(a)

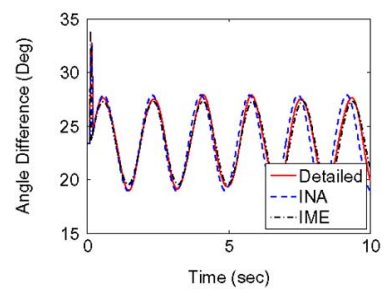

(c)

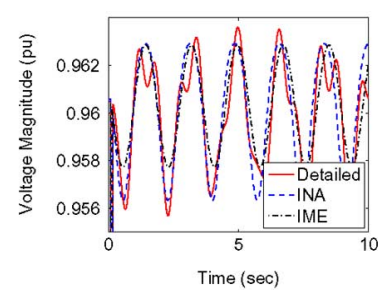

(b)

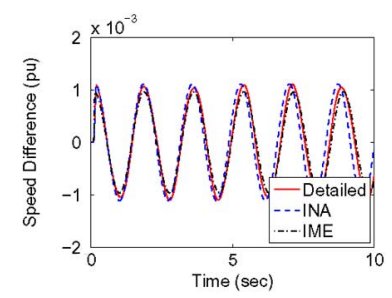

(d)
Fig. 10. Disturbance responses of the three models subject to three-phase faults on Bus 101. (a) Bus 3 voltage magnitude. (b) Bus 13 voltage magnitude. (c) Angle between Buses 3 and 13, $\Delta \theta=\theta_{3}-\theta_{13}$. (d) Machine speed difference $\Delta \omega=\omega_{1}-\omega_{2}$.

The disturbance is a three-phase short-circuit fault applied at Bus 101 in all three different models with $300 \mathrm{MW}$ of power transfer. No line is switched out, which would require using a different IME model. The impact of a three-phase short-circuit fault at Bus 101, which stops the power transfer between the two areas, can be quite different in these models. For the detailed model and the inertial aggregated model, the fault depresses the voltages at Buses 3 and 13, and reduces the loads on Buses 4 and 14. Thus, all the machine speeds in these two models will accelerate. On the other hand, in the IME model, $G_{2}$ is being supplied by the electrical power equal to the power transfer, and hence, its mechanical power is negative. As a result, when the fault is applied to Bus $101, G_{2}$ actually decelerates, whereas $G_{1}$ accelerates. As a result, for a fault of the same duration, the energy accumulated by the IME model will be higher than that of the detailed and inertia-aggregated models. Thus, to compare the disturbance response, we need to use different clearing times for the models.

Fig. 10 shows three traces: the responses of the detailed model and the inertial aggregated model to a five-cycle short-circuit fault on Bus 101, and the IME model to a two-cycle short-circuit fault on Bus 101. Note that the inertial aggregated model, which retains the loads on Buses 4 and 14, duplicates the interarea swing of the detailed model accurately. The IME model response also approximates the responses of the other two models quite well. Thus, the impact of a two-cycle short-circuit fault on Bus 101 applied to the IME model is about the same as a five-cycle fault on the same bus for the other two models. This is equivalent to saying that at the fault clearing time, the energies of these different models are approximately the same.

The disturbance response investigation here shows that the IME model can be used to assess system stability margins. In particular, the power-angle plot [20] of the reduced model in Fig. 2 can be constructed using the IME model and used to estimate critical clearing times and the impact of loss of transmission capacity.

\section{ApPliCATiOn to A TRANSFER PATH IN WECC}

In [8], synchronized phasor measurements from severe oscillation events were used to establish power-angle curves of

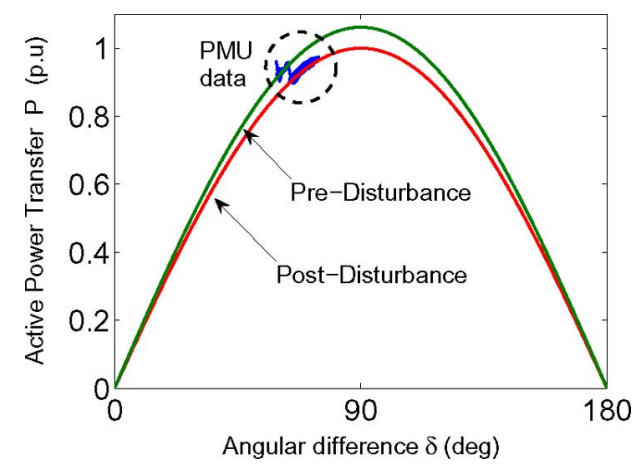

Fig. 11. Recomputed power-angle curves for Transfer Path 1.

two power transfer paths in WECC, without performing reactance extrapolation to the equivalent generator internal node. We apply the new techniques in this paper and recompute the IME model of Transfer Path 1 in [8], which is compensated by series capacitors. The resulting pre-disturbance and post-disturbance power-angle curves established from the new IME model are shown in Fig. 11, where the measured PMU data are highlighted. Compared to [8, Fig. 8], the new results here show much better agreement with actual system parameters.

\section{CONCLUSION}

In this paper, we have developed reactance and inertia extrapolation algorithms based on GPS-synchronized measurement data of bus voltage and line current phasors to estimate an equivalent interarea model of a two-area power system. This method can be used to represent the interarea dynamics across the power transfer interface of a multimachine power system exhibiting a single dominant mode of interarea oscillation. The use of the IME model to replicate the disturbance responses of the full model is demonstrated.

This work should be viewed as point of departure for several future investigations. The discussion in this paper does not include the effect of line charging and voltage control (for example, via a static var compensator) on the transfer interface, which will change the shape of the waveform in Fig. 5. The variation of the IME model reactance as a function of power transfer levels and the correlation of fault clearing times between the IME model and the detailed model need to be better understood. The applicability of this approach requires measuring interarea oscillations. For a well-damped power system such as the U.S. Eastern Interconnection, loss of a 1000-MW generating unit would normally induce oscillations lasting only two to three periods. Thus, good signal processing techniques need to be developed to extract the relevant modal response. Furthermore, the application of the method to large power systems with multiple interarea modes needs to be pursued.

\section{APPENDIX A \\ TWO-AREA SySTEM PARAMETERS}

A.1 Machine parameters on 900-MVA base: All the machines are classical models with identical parameters: $H=6.5$ p.u., $x_{d}^{\prime}=0.25$ p.u., $P_{m}=685 \mathrm{MW}$.

A. 2 Line Parameters on 100-MVA base:

$$
z_{1,10}=z_{11,110}=j 0.0167, z_{10,20}=z_{110,120}=j 0.025
$$


$z_{2,20}=z_{12,120}=j 0.0167, z_{20,3}=z_{120,13}=j 0.01$

$z_{3,4}=z_{13,14}=j 0.005, z_{3,101}=z_{101,13}=0.0055+$ j0.055

A.3 Loads $-300 \mathrm{MW}$ power transfer: $L_{4}=10.7 \mathrm{MW}$ and $L_{14}=16.7 \mathrm{MW} ; 450 \mathrm{MW}$ power transfer: $L_{4}=9.2 \mathrm{MW}$ and $L_{14}=18.2 \mathrm{MW} ; 150 \mathrm{MW}$ power transfer, $L_{4}=12.2 \mathrm{MW}$ and $L_{14}=14.9 \mathrm{MW}$.

\section{APPENDIX B \\ TWO-MACHINE SYSTEM PARAMETERS}

B.1 Machine parameters on 900-MVA base: $H_{1}=6.5$ p.u., $H_{2}=9.5$ p.u., $x_{l 1}=x_{l 2}=0.20$ p.u., $x_{d 1}=x_{d 2}=1.80$ p.u., $x_{d 1}^{\prime}=0.25$ p.u., $x_{d 2}^{\prime}=0.30$ p.u., $x_{d 1}^{\prime \prime}=0.20$ p.u., $x_{d 2}^{\prime \prime}=0.25$ p.u., $\tau_{d 0_{1}}^{\prime}=\tau_{d 0_{2}}^{\prime}=8.0 \mathrm{~s}, \tau_{d 0_{1}}^{\prime \prime}=\tau_{d 0_{2}}^{\prime \prime}=0.03 \mathrm{~s}, x_{q_{1}}=$ $x_{q_{2}}=1.7$ p.u., $x_{q_{1}}^{\prime}=x_{q_{2}}^{\prime}=0.55$ p.u., $x_{q_{1}}^{\prime \prime}=x_{q_{2}}^{\prime \prime}=0.24$ p.u., $\tau_{q 0_{1}}^{\prime}=\tau_{q 0_{2}}^{\prime}=0.4 \mathrm{~s}, \tau_{q 0_{1}}^{\prime \prime}=\tau_{q 0_{2}}^{\prime \prime}=0.05 \mathrm{~s}$.

B.2 Line parameters on 100-MVA base: $x_{e}=0.055$ p.u., $x_{T 1}=0.01$ p.u., $x_{T 2}=0.01$ p.u.

B.3 Excitation System Parameters-Model Type AC4A [16]: $T_{A}=0.015 \mathrm{~s}, T_{B}=10 \mathrm{~s}, T_{C}=1 \mathrm{~s}, K_{A}=100$.

\section{ACKNOWLEDGMENT}

The authors would like to thank B. Bhargava and A. Salazar from Southern California Edison for their technical discussion of this work.

\section{REFERENCES}

[1] R. W. de Mello, R. Podmore, and K. N. Stanton, "Coherency-based dynamic equivalents: Applications in transient stability studies," in Proc. PICA Conf., 1975, pp. 23-31.

[2] A. J. Germond and R. Podmore, "Dynamic aggregation of generating unit models," IEEE Trans. Power App. Syst., vol. PAS-97, no. 4, pp. 1060-1069, Jul. 1978

[3] J. H. Chow, G. Peponides, P. V. Kokotovic, B. Avramovic, and J. R. Winkelman, Time-Scale Modeling of Dynamic Networks with Applications to Power Systems. New York: Springer-Verlag, 1982.

[4] J. H. Chow and P. V. Kokotovic, "Time scale modeling of sparse dynamic networks," IEEE Trans. Autom. Control, vol. 30, no. 8, pp. 714-722, Aug. 1985.

[5] R. Date and J. H. Chow, "Aggregation properties of linearized twotime-scale power networks," IEEE Trans. Circuits Syst., vol. 38, no. 7, pp. 720-730, Jul. 1991

[6] A. G. Phadke, J. S. Thorp, and M. G. Adamiak, "New measurement techniques for tracking voltage phasors, local system frequency, and rate of change of frequency," IEEE Trans. Power App. Syst., vol. PAS102, no. 5, pp. 1025-1038, May 1983.

[7] A. G. Phadke, "Synchronized phasor measurements in power systems," IEEE Comput. Appl. Power, vol. 6, no. 2, pp. 10-15, Apr. 1993.

[8] J. H. Chow, A. Chakrabortty, M. Arcak, B. Bhargava, and A. Salazar, "Synchronized phasor data based energy function analysis of dominant power transfer paths in large power systems," IEEE Trans. Power Syst., vol. 22, no. 2, pp. 727-734, May 2007.

[9] V. Centeno, A. G. Phadke, A. Edris, J. Benton, M. Gaudi, and G. Michel, "An adaptive out-of-step relay for power system protection," IEEE Trans. Power Del., vol. 12, no. 1, pp. 61-71, Jan. 1997.

[10] W. R. Roemish and E. T. Wall, "A new synchronous generator out-ofstep relay scheme, part I,' IEEE Trans. Power App. Syst., vol. PAS-104, no. 3, pp. 562-571, Mar. 1985.
[11] J. Hauer, D. Trudnowski, G. Rogers, B. Mittelstadt, W. Litzen-Berger, and J. Johnson, "Keeping an eye on power system dynamics," IEEE Comput. Appl. Power, vol. 10, no. 4, pp. 50-54, Oct. 1997.

[12] J. R. Smith, F. Fatehi, C. S. Woods, J. F. Hauer, and D. J. Trudnowski, "Transfer function identification in power system applications," IEEE Trans. Power Syst., vol. 8, no. 3, pp. 1282-1290, Aug. 1993.

[13] D. N. Kosterev, C. W. Taylor, and W. A. Mittelstadt, "Model validation for the August 10, 1996 WSCC system outage," IEEE Trans. Power Syst., vol. 14, no. 3, pp. 967-979, Aug. 1999.

[14] M. Klein, G. J. Rogers, and P. Kundur, "A fundamental study of interarea oscillations in power systems," IEEE Trans. Power Syst., vol. 6 , no. 3, pp. 914-921, Aug. 1991.

[15] G. Rogers, Power System Oscillations. Norwell, MA: Kluwer, 1999.

[16] IEEE Recommended Practice for Excitation System Models for Power System Stability Studies, IEEE Std. 421.5, Aug. 1992.

[17] J. H. Chow and K. W. Cheung, "A toolbox for power system dynamics and control engineering education and research," IEEE Trans. Power Syst., vol. 7, no. 4, pp. 1559-1564, Nov. 1992.

[18] J. J. Sanchez-Gasca and J. H. Chow, "Performance comparison of three identification methods for the analysis of electromechanical oscillations," IEEE Trans. Power Syst., vol. 14, no. 3, pp. 995-1001, Aug. 1999.

[19] I. Kamwa, R. Grondin, E. J. Dickinson, and S. Fortin, “A minimal realization approach to reduced-order modelling and modal analysis for power system response signals," IEEE Trans. Power Syst., vol. 8, no. 3, pp. 1020-1029, Aug. 1993.

[20] A. R. Bergen and V. Vittal, Power System Analysis, 2nd ed. Englewood Cliffs, NJ: Prentice-Hall, 1999.
Joe H. Chow (F'92) received the B.S.E.E. and B.Math. degrees from the University of Minnesota, Minneapolis, and the M.S. and Ph.D. degrees from the University of Illinois, Urbana.

$\mathrm{He}$ is a Professor with the Electrical, Computer, and Systems Engineering Department at Rensselaer Polytechnic Institute, Troy, NY. His current research interests include modeling and control of power systems, FACTS controllers, and synchronized phasor measurements.

Aranya Chakrabortty (S'06) received the B.E. degree from Jadavpur University, Calcutta, India in 2004 and the M.S. degree from Rensselaer Polytechnic Institute (RPI), Troy, NY, in 2005, both in electrical engineering. He pursuing the Ph.D. degree in the Electrical, Computer, and Systems Engineering Department at RPI.

His research interests are in control systems.

Luigi Vanfretti (S'03) received the B.S.E.E. degree from the San Carlos University of Guatemala, Guatemala City, in June 2005. He is pursuing the M.S./Ph.D degree in the Electrical, Computer, and Systems Engineering Department at Rensselaer Polytechnic Institute, Troy, NY.

His research interests are dynamics, stability, control, and security of power systems.

Mr. Vanfretti received the Francisco Vela award in February 2006.

Murat Arcak (SM'05) received the B.S. degree in electrical and electronics engineering from the Bogazici University, Istanbul, Turkey, in 1996 and the M.S and Ph.D. degrees in electrical and computer engineering from the University of California, Santa Barbara, in 1997 and 2000, respectively, under the direction of P. Kokotovic.

He is an Associate Professor in the Electrical, Computer, and Systems Engineering Department at the Rensselaer Polytechnic Institute, Troy, NY. His research is in nonlinear control theory and its applications. 